\title{
THE PHENOMENON OF CULTURAL HERITAGE AND THE DEFINITION OF A UNIT OF MATERIAL
}

\section{Ivo Maroevic}

Cultural heritage is a complex matter. To use a very concise definition, it is the value of the past that we distinguish in the present in order to be able to preserve it for the future. Through the varying course of the present it constantly transmits the experiencees and the messages of past times, forever expanding human knowledge about them. The theoretical formulation of cultural heritage goes back a long way but in the modern sense began to be defined and directed towards conservation in the period of romanticism, in the mid-19th century, when a clearly focused interest in the past was one of the major features of the contemporary world-view.

Since then, cultural heritage has become a phenomenon of expanding dimensions. The way in which it is used and presented has been transformed so that it now constitutes a part of social reality, of diverse intensity and scope in differing social groups and classes, as well as among those individuals who have a sense of its cultural intent.

On the other hand, cultural heritage is also a witness of and testimony to time, space and society, the three key determinants of the environment in which we live.

The course of time leaves its traces on all the material and semantic components of cultural heritage. Material, because the passage of chronological time together with environmental influences give rise to the ageing and degradation of the material of which cultural heritage is constructed. In addition, the context in which objects exist cause both deliberate and unintentional changes in the appearance, structure and significance of the world of objects that surrounds us. This very fact makes the material and form of the objects of cultural heritage witness to the passage of this time.

Space is the framework in which most of the material world that we have recognised as cultural heritage lives. It changes, through both the modification of the natural into a cultivated landscape and the construction of communications and power plants, the development of settle- 
136 ments and economic centres. Space is the physical context of the occurrence of cultural heritage, with which people often identify themselves, and it often becomes an important factor in the spiritual context. A change in context also often changes the significance of cultural heritage. In their materialised form, objects physically exist in real space. This does not mean that objects have never been transmitted from one segment of space to another; however, most material cultural heritage has a powerful bond with the space in which it originated and in which it exists.

It is in society that cultural heritage brings to bear its meaning, although on the other hand society has an effect on the semantic context of the way in which cultural heritage is understood and experienced. For this reason the social environment is the most complex, as the significance of cultural heritage is experienced as a symbiosis of place and time. Thus cultural heritage will transmit its significance in a society through the agency of materials and forms but often, too, in a virtual way thereby becoming a part of virtual reality. In parallel, during its life in diverse historical circumstances it will add further layers to its own meaning which may not necessarily be inscribed in its physical structure but will create a kind of aura that will have an effect on the distinctiveness of its significance in each of its presents.

It is actually the concord of material, form and significance, which is manifested in time, space and society, that makes individual parts of cultural heritage authentic; the concept of authenticity in this context not being limited to the question of origin, but including significances and changes that have gathered in the material and spiritual component of cultural heritage throughout its existence.

Cultural heritage can be both material and non-material. This is a basic division in which, if we make use of a diagram, the field of the non-material or spiritual heritage encompasses that of the material (Fig. 1). This does not imply, as might at first appear, the domination of the material by the spiritual, but the recognition that that part of the cultural heritage which is expressed in words, music or movement is neither materialised nor available to other people and generations except through a person who interprets, or a medium which gives substance to, just one of its possible variants. The part that is materialised in human hand-work (in the widest possible meaning of the word) has in its form and material, or in the significance associated with them, a mass of spiritual, non-material values and messages, which are much more difficult to read and understand, for the medium of the object is unconventional, rationally unreadable to many, and yet emotionally close. Cultural heritage is a phenomenon that in a very specific way affects and purposefully ties together human rational and emotional components.

Material cultural heritage, in addition, is divided according to fundamental features into immovable and movable. The immovable is firmly linked to a given space, it is subject to various destructive influences, both natural and those brought about by human beings through their deliberate and unintentional actions. As a rule the movable heritage is less dependent on its spatial location. It can change contexts 


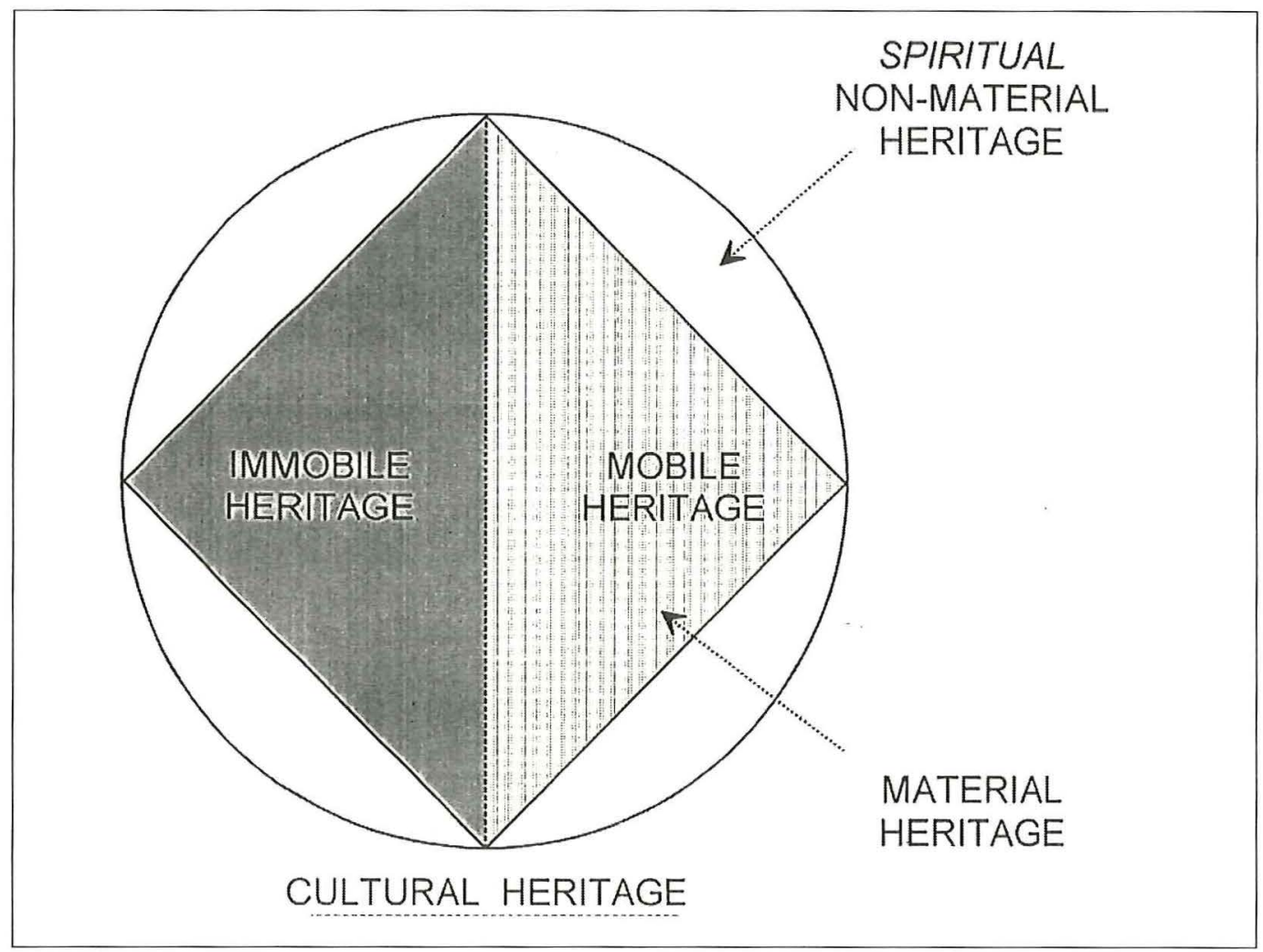

Fig. 1: Relations of material and non-material heritage within the structure of cultural heritage.

and owners, and it is possible for people to look after it much more effectively, should they want to, for it is not necessary to allow the ravages of nature to affect it. It is primarily looked after in indoor spaces, and can more easily be moved and taken to a safer place, although a change of context can alter its primary meaning, as well as some subsequently acquired meanings.

Movable cultural heritage is usually kept in museums (as long as we understand all kinds of collections that are not institutionalised as also being museums), archives and libraries. Its material component is preserved and used for the study and transmission of messages, as are those forms of non-material cultural heritage which have been materialised in a given moment of time, as some kind of documentation of their existence. In these institutions various forms of documentation of cultural heritage are preserved and processed. If we recall that the documentation of cultural heritage is an organised process of registration of information that objects and whole units of cultural heritage possess and emit, then we will agree that research into objects and the processing of them are the foundation of docu- 
IVO MAROEVIC

138 mentation. This process is based on data about an object or a whole, the number and quality of which are defined by some standard or convention, so that they can afford us as accurate an idea as possible about the object or the whole, from all the professional and scientific aspects of the time in which the documentation is being drawn up (Maroevic, 1993:190). On the other hand documentation is a medium in which knowledge about heritage is formatted. The different materials, forms and formats, and thus characters of media with whose help we document diverse forms of the treatment of objects of cultural heritage, turn documentation into a corpus which is worth dealing with and which at a given moment can replace the original object. The diversity of the material gathered, handled and used in museums, archives and libraries affects their appearances and the organisation of their work. Museums, in this series of institutions, are in a border area, for they collect objects as sources of information and knowledge that as a rule are not transferred to any of the more transparent media for the purpose of communication. In archives it is mainly unique documents that are collected on paper and in media that register text and image; these documents attend, and thus indirectly bear witness to, events and the lives of people and their societies. Libraries use books that condense human thinking and knowledge into set formats, and serve, or served, the spread of knowledge and messages transposed into the medium of the written or printed word. Books also transmit a majority of the statements and messages of art, which quite often in themselves represent cultural heritage.
Because of the particularity of the tasks and the way these three institutions act, we could interpret their differences according to the model of the museological interpretation of the museum object (Fig. 2), making use of the basic semiological analysis of the sign. The sign can be separated into its component parts: signifier $(\mathrm{Sr})$, sign $(\mathrm{Sn})$ and signified $(\mathrm{Sd})$. Analogously, the cultural heritage object can be separated into its three component parts: material (M), form (F), and significance (S). Depending on how far the object has shifted away from the reality that it represents we could say that all three of these components are equally important in the interpretation and determination of the value of the museum object, with the material most faithfully reflecting the existence of the object in time, the form being most effective in the process of disseminating the message in space, and the meaning of the object penetrating society in the shape of information. Archival material relies more on material and meaning, while form, which states that meaning, can be almost equally valuably stored and used in some other medium. This does not mean that form is unimportant, but that to a large extent it can be replaced by a faithful copy. Archival material can also be used via a medium, so that the original material and its associated meaning can be better preserved. If we leave aside for the moment particularly valuable old books that have the significance of museum objects, in most libraries the meaning that is contained in books is dominant. Their material and form are also important and valuable, but not to the same extent. This is especially true since, after the invention of prin- 
ting, books have been published in large numbers of copies, and the possibilities of reprints and new editions have reduced the aspiration of preserving the original materials and forms of books. The museum object in the virtual museum, too, will reduce the role of materials in the experience of its meaning. It will trust everything to form and the communicated significance.

This simplified analysis of museum, archival and library materials is neither presented nor conceived in order to reduce the importance of individual kinds of material nor to suggest that archives or libraries do not have to take care of the original relations of material, form and meaning in their stocks. It simply attempts to show that in all activities that deal with movable cultural heritage, not all the material or formal components of the units of material or the stocks that are preserved and used have the same specific gravity regarding the communication of the messages that they contain.
Thus the definition of a unit of material can be posited on a very general basis, and then, if we imagine it in graphic form, broadened pyramidally towards the base, using in the process the model of the three sided pyramid, the base of which is made up of archives, libraries and museums (Fig. 3). All these three activities work in a reality that we can provisionally designate controlled reality. Controlled because the people who work in these institutions can control the life and use of the objects (the material) that they look after. All of them, in other words, can provide a certain kind of care for their stocks; they can exclude the likelihood that they will decay because of the action of external factors, whether natural or social. Stocks in these institutions are not at any risk from society, nor is there any chance that they may find themselves in a group of objects that have become socially obsolescent and rejected. The small percentage of surplus and damaged items that are discarded during use does not refute this statement, al-
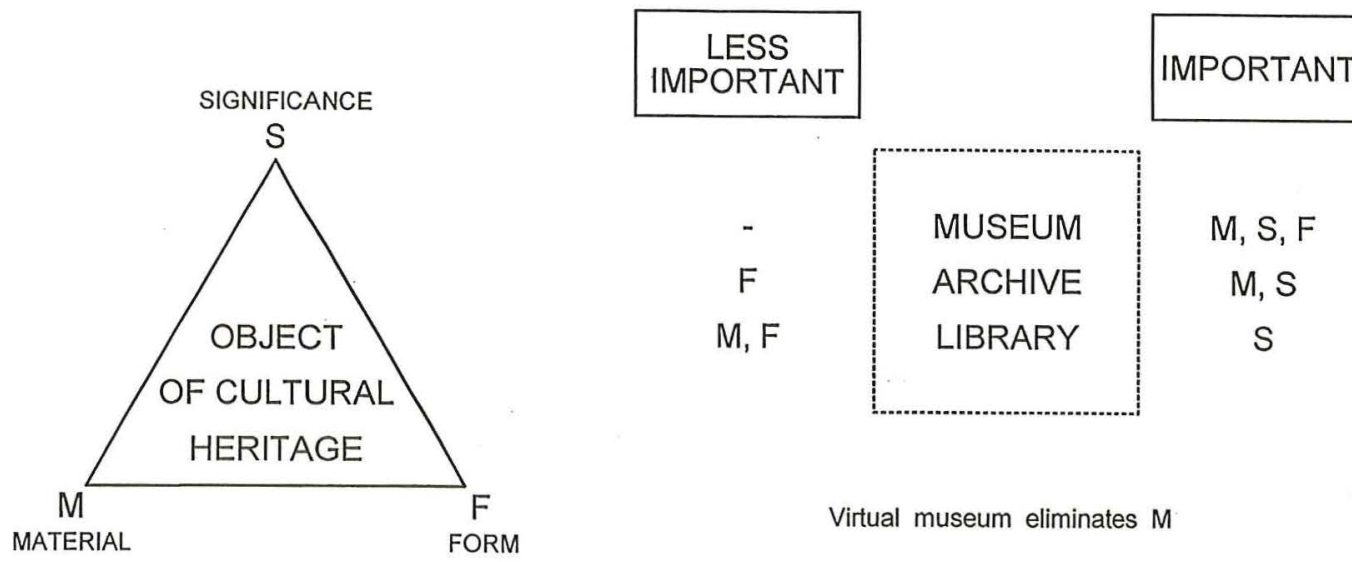

Fig. 2: Stages of importance of the components of cultural heritage objects in museums, archives and libraries. 
IVO MAROEVIC

140 though in the discarding process the model of diverse attitudes with respect to materi$\mathrm{al}$, form and significance in connection with a museum object, an archival document or a book could well be applied.

Museums constitute the lowest level of this irregular pyramid. They are at the border of where controlled and genuine reality meet, for objects come into a museum by being separated out from their primary or archaeological context, and transferred to the museum context, where they assume the aura of cultural heritage. Archives are on a somewhat higher level of abstraction in relation to the real world. They too collect material that developed in genuine reality, but the material is already defined and structured according to media. The material reflects the social context in which it came into being and bears witness to almost all the events registered, being reflection of them in a medium. Libraries are at the upper boundary of controlled reality. Their material is part of the system of the world of objects, but contains a formulated or, perhaps, a formatted knowledge and artistic statements at a somewhat higher level of abstraction. A book is not a medium that comes into being like that part of social reality in which history and living practice might accumulate, but contains formatted knowledge or artistic expressions which, through their multiple dissemination in time and space, also play an exceptionally important social role. Libraries are on the boundary line between controlled and virtual reality.

If we observe the direction of these three activities or institutions of which we are speaking, then we shall see that the world of objects becomes more and more abstract as we proceed from the museum towards the library, becoming more and more a reflection of reality rather than reality itself. In contrast we can see that documentation descends towards reality as we go from the library to the museum. An object in a museum can be seen and touched. The life of the same object can be followed in archival documents, in the evidence of events in which it took part, at the level of the unique document. The same object can be seen in a picture in a book, where we can also read everything that people have found out about it. Synthesis is still more abstract here, for it has parted company completely with the life of the object, although it does, from its abstract level, still indirectly affect it. I cannot refrain from quoting Zeljka Corak, who gives an excellent summary of the process in terms of a cathedral in Cambrai, which was destroyed in the 18 th century; a model of the cathedral was in turn destroyed leaving behind only a photograph. Hence the existence of the cathedral moved:

from the body to the sign, from the sign to the likeness, dependent on ever more indirect indicators. Ever more fragile buttresses supported ever more air. (Corak, 1991:64)

Information, irrespective of whether it is concealed in a museum, an archive or a library, i.e. in the part of the world of objects that is looked after in these institutions, lives in virtual reality. It is part of the common information environment and only here can we talk of an effective collaboration at the level of information among those institutions which work in controlled reality. We must not overlook 
Fig. 3: Virtual reality in relation with other realities in which museums, archives and libraries act as information sources.

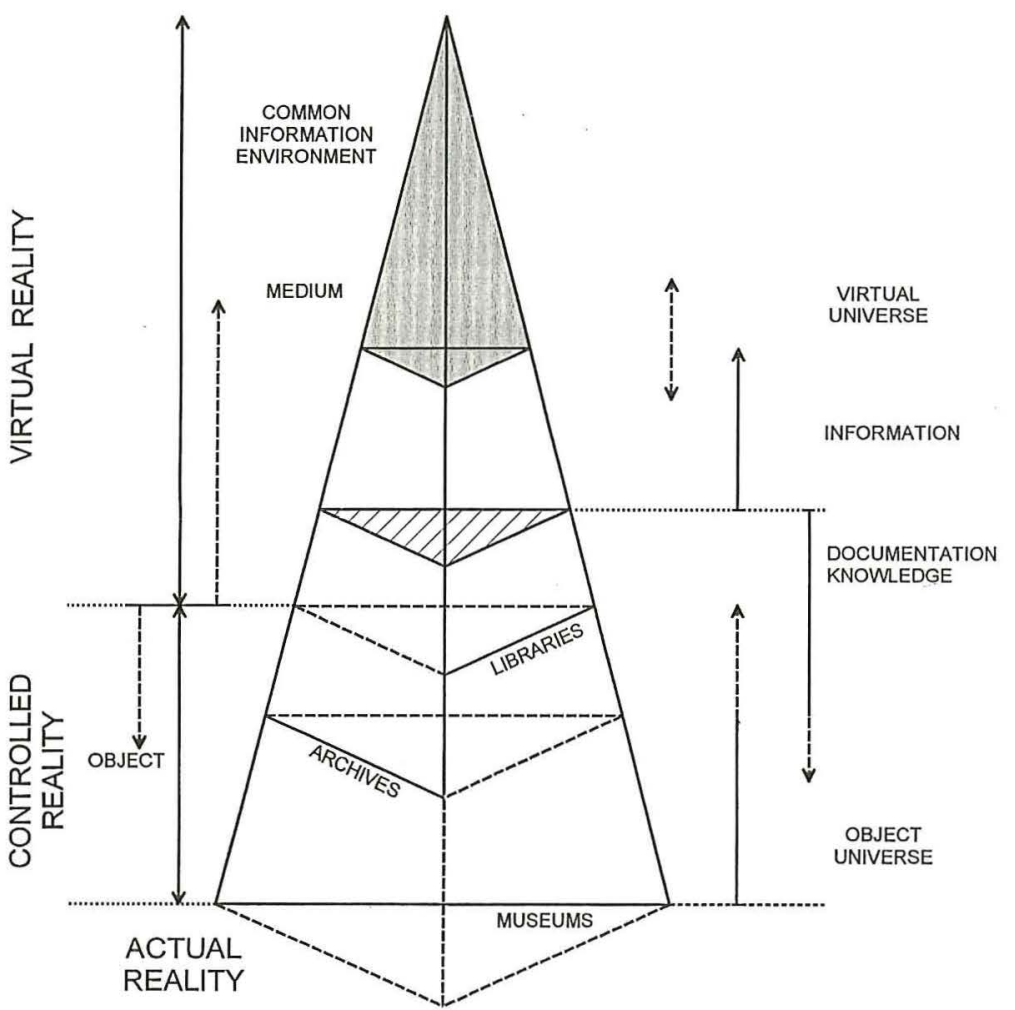

information accessible to all has also created a problem about selectivity, about how to cope with the enormous amount of insufficiently evaluated and retrievable data. The structures that are closest to reality are the most complex, for in them there is a considerably greater amount of structural information. Structures that are brought to a certain degree of abstraction, because they use the media or are media themselves, reduce information to a manageable amount, so that it does not threaten the selective approach to the other kind of information. Ideas that ever more sophisticated forms of aids or tools will help us to select relevant information from the fact that in information theory two tion, that we will be able to store it in computers and data banks, where it can be searched and retrieved. With structural information the processes will be more complex and it will not always be possible to fit it into the classic reserves of information which we can count and rely on. This virtual world of information, which today, thanks to the great world networks, has made an enormous amount of 
142 the abundance that fills the information networks and documentation systems every day are completely beyond the point. They will simply make the work easier, while choice will always be up to people, who will input their own criteria and the individuality of their own intuition into complex information systems. This is particularly important for information systems related to the social sciences or arts, in which the final choice of information will never be able to make complete use of the benefits of the information infrastructure. In other words, in the selection of information, computers will never be able to replace people.

In this context, the unit of material might be the object or a set of objects of the material culture which, qua cultural heritage, are used and researched into in one of the institutions from the lower part of the pyramid, irrespective of the level of abstraction regarding the world of objects the given institution deals with. At the same time the unit of documentation material can be considered to be, and defined as, the set of documentation data that will be able entirely and correctly to represent the object or set of objects of the material of the museum, archive or library. The compatibility of such a set of data will vary, depending on how far it is from the apex or the base of the pyramid mentioned earlier (Fig. 3). The closer we are to the apex, the more the data obtained at the lower levels will be able to be used universally, with the proviso that the degree of imprecision with respect to the object we are dealing with will be greater and greater. The advantage of this global information environment, in which data and information created at the levels of genuine and controlled reality become a part, lies in the fact that in a virtual reality created in this way various fields and areas of human activity can be brought together, as can various forms of documentation and the media in which they are stored; allowing their physical existence, their weaknesses and drawbacks to be ignored. We then experience them as part of the world that lies somewhere outside us, at a conceptual level. From this conceptual level we can descend to the level of genuine or controlled reality with completely different understandings about the relations between items of information and about the objects they refer to, applying some other, hitherto unguessed at forms and combinations of knowledge. Here unimagined new challenges arise, challenges that are outside the scope of this time and this discussion.

\section{LITERATURE}

Corak, Zeljka. Krhotine (Fragments), Zagreb, Graficki zavod Hrvatske, 1991

Maroevic, Ivo. Uvod u muzeologiju (Introduction to Museology), Institute for Information Studies, Faculty of Philosophy, University of Zagreb, 1993

Tudjman, Miroslav. Struktura kulturne informacije (The structure of cultural information). Zagreb, Institute for the Culture of Croatia, 1983.

Prof. Dr Ivo Maroevic is Chairperson, Chair of Museology, Information Science Department, Faculty of Philosophy, Zagreb University Adr: Miramarska 134, 10000 Zagreb, Croatia Fax: $+385-16156879$ 